\title{
呼吸图法制备基于准聚轮烷的响应性薄膜
}

\author{
董锦辉李进杰王赫刘涁秀彭博陈健壮* 林绍梁 \\ (华东理工大学 材料科学与工程学院 上海 200237)
}

\begin{abstract}
摘要 响应性薄膜能够响应外界的刺激来改变自身的结构或性能, 是智能材料的重要组成部分. 本工作以 $1,4-二 乙$ 氧 基柱[5]芳烃(1,4-diethoxypillar[5] arene, DEP5A)和聚己内酯- $b$-聚乙二醇- $b$-聚己内酯(PCL- $b$-PEG- $b$-PCL)构建的准聚轮烷 (polypseudorotaxane, PPR)为原料, 利用呼吸图法制备出了蜂窝状多孔及球状组装体的疏水薄膜. 研究发现溶剂、浓度、 柱芳烃物质的量比和成膜气氛等因素对薄膜表面形貌均能产生较大的影响. 通过制膜条件的优化, 在水汽氛围中制备 出了表面具有规则蜂窝状结构的多孔薄膜, 在乙醇氛围下制备出了表面为规整的褶皱球状组装体的薄膜. 研究表明, 上述两种具有规则表面形貌的 PPR 薄膜对竞争性客体 1,4-二溴丁烷都具有响应性，表现出薄膜表面形貌的变化及亲疏 水性的变化. 此类响应性薄膜在微量液体无损转移、功能性涂层和智能薄膜等方面具有潜在的应用前景.
\end{abstract}

关键词 准聚轮烷; 柱芳烃; 呼吸图法; 响应性薄膜; 疏水性

\section{Fabrication of Polypseudorotaxane-Based Responsive Film via Breath Figure Method}

\author{
Jinhui Dong Jinjie Li He Wang Binxiu Liu Bo Peng \\ Jianzhuang Chen* Shaoliang Lin
}

(School of Materials Science and Engineering, East China University of Science and Technology, Shanghai 200237, China)

\begin{abstract}
Stimuli-responsive film is an essential part of smart materials for its manipulation of structures or performances in response to external stimulations, which is crucial to the exploitation of actuators, sensors, and soft robotics. Herein, a novel polypseudorotaxane (PPR) with competitive guest-responsiveness was leveraged to fabricate polymer films with diverse surface morphologies via breath figure (BF) method under water or ethanol atmosphere. PPR was constructed based on the selective recognition of 1,4-diethoxypillar[5]arene (DEP5A) to the PCL block of polycaprolactone-block-poly(ethylene glycol)-block-polycaprolactone (PCL- $b$-PEG- $b$-PCL) in $\mathrm{CHCl}_{3}$. The morphologies of such polymer films were observed by scanning electron microscopy (SEM). Specifically, ordered honeycomb porous films with an average pore size of approximately $2.15 \mu \mathrm{m}$ and sphere films with an average diameter of approximately $3.34 \mu \mathrm{m}$ were fabricated via BF method under water atmosphere and ethanol atmosphere, respectively. The influences of solvent, concentration, and the equivalent ratio of DEP5A to PCL- $b$-PEG- $b$-PCL on the nanostructures of films were investigated. It was found that the films with ordered microstructures were obtained using PPR (15.0 equiv. of DEP5A to PCL- $b$-PEG- $b$-PCL, $5.0 \mathrm{mg} \cdot \mathrm{mL}^{-1}$ of PCL- $b$-PEG- $b$-PCL) solution under a constant relative humidity of $90 \%$ or ethanol atmosphere at $25{ }^{\circ} \mathrm{C}$. These films showed hydrophobic (water contact angle over $130^{\circ}$ ) due to their hierarchical structures. Furthermore, the obtained films were immersed in competitive guest-containing solution $(20.0 \mu \mathrm{L} \mathrm{DBrBu}+10.0 \mathrm{~mL}$ ethanol) to manipulate their structures and hydrophobicity. The competitive guest, 1,4-dibromobutane (DBrBu), showed stronger affinity than PCL block to DEP5A. DEP5A would be detached from the PCL block and form pseudorotaxane with $\mathrm{DBrBu}$ after immersing the films in competitive guest-containing solution. With the immersion time increasing, PPR were destroyed progressively, resulting in the formation of cuboid-crystal films and the decrement of water contact angle. This result provided a facile strategy to fabricate a smart film with controllable wettability and to explore the potential application prospects in the non-destructive transfer of trace liquids, functional coatings and smart film materials.
\end{abstract}

Keywords polypseudorotaxane; pillararene; breath figure method; responsive film; hydrophobicity

\section{1 引言}

响应性薄膜能够响应外界的刺激来改变自身的结 构或性能, 研究者通过改变光、 $\mathrm{pH}$ 、温度、机械力等外
界环境因素, 使薄膜发生形状、结构和性能等方面的改 变, 拓宽薄膜在驱动器、传感器和软体机器人等应用场 景 ${ }^{[1-9]}$. 润湿性是薄膜的重要表面性质, 受大自然的启 发, 研究者制备出了如超疏水低粘附表面、超疏水高粘

\footnotetext{
* E-mail: chenjianzhuang@ecust.edu.cn
}

Received March 22, 2021; published May 11, 2021.

Supporting information for this article is available free of charge via the Internet at http://sioc-journal.cn.

Project supported by the National Natural Science Foundation of China (Nos. 52073092, 51873061, 21404039), and the Natural Science Foundation of Shanghai (No. 18ZR1408200).

项目受国家自然科学基金(Nos. 52073092, 51873061, 21404039)和上海市自然科学基金(No. 18ZR1408200)资助. 


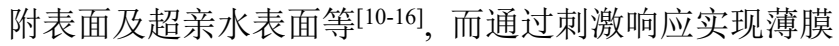
润湿性的变化, 则可以实现薄膜在不同应用领域的跨 越 ${ }^{[17-21]}$. 准聚轮烷(polypseudorotaxane, PPR)是主体环状 分子和客体线型高分子在非共价键作用下形成的新型 超分子体系 ${ }^{[22-24]}$. 动态可逆的主客体作用使得准聚轮烷 具有热、 $\mathrm{pH}$ 和竞争性客体响应 ${ }^{[25-30]}$. 各种类型的主体分 子, 例如冠醚、环糊精、杯芳烃、葫芦脲和柱芳烃等已 被用于制备多种拓扑结构的准聚轮烷. 因此, 准聚轮烷 在制备响应性薄膜的材料方面展现出良好的应用前 景[31-36].

呼吸图法是指聚合物溶液在高湿度环境下挥发, 溶 液表面温度迅速降低, 水滴冷凝下来并在毛细作用下形 成六方堆积阵列, 随着溶剂和水的挥发, 最终获得微米 级规整多孔薄膜 ${ }^{[37-38]}$. 通过改变气氛成分, 例如乙醇或 甲醇氛围, 利用气氛冷凝成的液体与所用溶剂表面张力 的差异, 则能制备出球状等组装体薄膜. 本课题组近年 来研究光控形变多孔薄膜及球状组装体薄膜, 实现了薄 膜微米级孔和球形状的精确控制及薄膜润湿性改 变 ${ }^{[39-43]}$. 通过不断的研究, 不同因素对多孔薄膜结构的 影响以及成孔的机理已经取得了较大的进展 ${ }^{[44]}$.

本文中, 我们采用简便、快速和低能耗的呼吸图方 法用 PPR 制备了疏水的超分子薄膜. PPR 的构建是基于 1,4-二乙氧基柱 [5] 芳烃 (1,4-diethoxypillar[5]arene, DEP5A) 与聚已内酯 $-b$ - 聚乙二醇 $-b$ - 聚己内酯 (PCL- $b$-PEG- $b$-PCL) 中的 PCL 链段在 $\mathrm{CHCl}_{3}$ 中的选择性 络合, 这种选择性络合能通过加入竞争性客体 1,4-二溴 丁烷 (1,4-dibromobutane, $\mathrm{DBrBu})$ 来解离, 这是由于 $\mathrm{DBrBu}$ 与 $\mathrm{DEP} 5 \mathrm{~A}$ 具有更强的主客体作用并形成准轮烷. 在水和乙醇氛围中, 用 PPR 分别制备了蜂窝状多孔薄膜
和球状组装体薄膜，这两种薄膜都展现出疏水性. 进一 步，将两种薄膜浸入含竞争性客体的乙醇溶液中，薄膜 微结构发生变化, 逐渐形成片状组装体结构. 特别的是, 随着结构的逐渐改变, 薄膜的疏水性逐渐减弱. 通过简 单的制备方式和材料的响应性，我们最终制备了疏水的 超分子多孔和球状组装体薄膜并实现了薄膜微结构及 润湿性的调控.

\section{2 结果与讨论}

如图 1 所示, 本文构建了一种新型的 PPR. 在氯仿 中, 基于 DEP5A 选择性络合 PCL- $b$-PEG- $b$-PCL 中的 PCL 链段, 形成了一种具有竞争性客体响应的新型 $\mathrm{PPR}^{[43]}$. 采用呼吸图的制备方法, 在不同气氛下将其滴 在玻璃基板上, 可以形成具有不同微观形貌的薄膜.

\section{1 呼吸图法制备有序的 PPR 超分子薄膜}

形成规整的呼吸图阵列主要取决于两大基本要素: 聚合物的特性和环境因素. 我们探索了 PPR 形成规整形 貌薄膜的最佳条件并测试了薄膜的润湿性, 对比了纯聚 合物与 PPR 在相同条件下制备薄膜的表面形貌.

\section{1 .1 水汽氛围下制备 PPR 蜂窝状多孔薄膜}

如图 1a 所示, 多孔薄膜的形成主要分为 3 个阶段: (1) PPR 溶液滴在玻璃片上, 在高湿度环境下, 溶剂快速 挥发, 溶液表面温度迅速降低, 水汽冷凝, 由于水具有 高表面张力, 因此在溶液表面形成水滴; (2)溶剂进一步 挥发, 水滴由于毛细作用力, 在溶液表面形成六方堆积 阵列; (3)溶剂和水滴逐渐挥发, 由于水滴的沉降作用, 最终形成了蜂窝状多孔结构的薄膜.

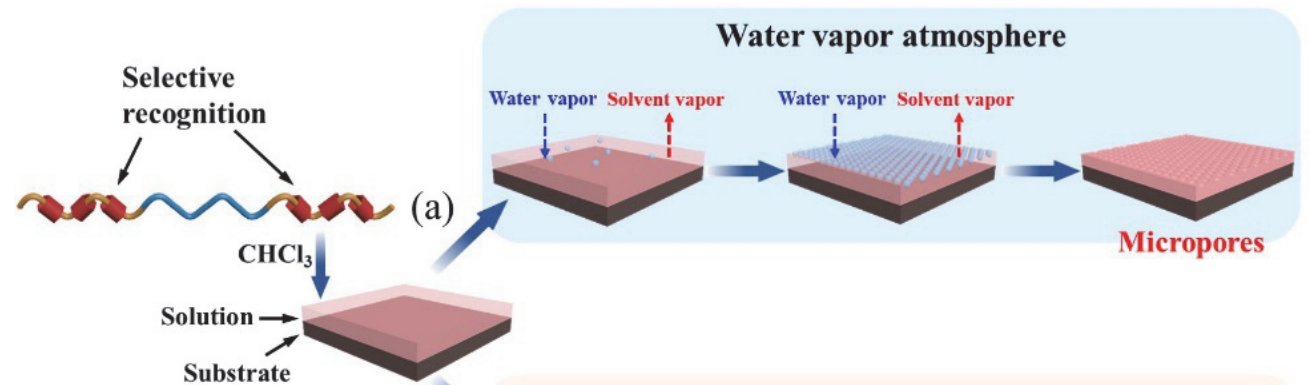

(b)

Ethanol vapor atmosphere

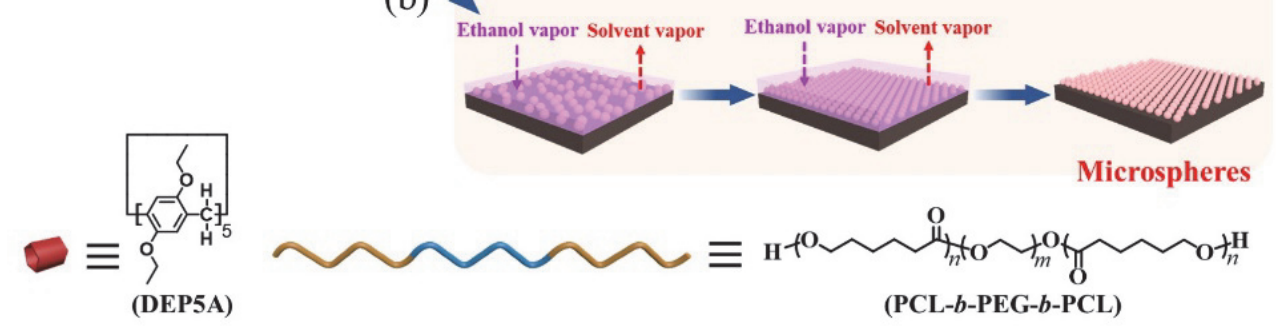

图 1 PCL- $b$-PEG- $b-$ PCL 与 DEP5A 通过主客体作用在氯仿中形成准聚轮烷并在(a)水汽氛围中形成蜂窝状多孔薄膜和在(b)乙醇氛围中形成球状组 装体薄膜的示意图

Figure 1 Cartoon representation of the construction of PPR based on the host-guest interaction between PCL- $b$-PEG- $b$-PCL and DEP5A in CHCl ${ }_{3}$ and the PPR solution was used to fabricate (a) honeycomb porous films under water vapor atmosphere and (b) sphere films under ethanol vapor atmosphere 
我们探索了成膜物质种类、溶剂、湿度、浓度和柱 芳烃物质的量比等对形成多孔薄膜的影响(如图 $\mathrm{S} 1 \sim$ S5), 发现在 $25{ }^{\circ} \mathrm{C}$ 和 $90 \% \mathrm{RH}$ 的条件下, 在不加入柱芳 烃的情况下, 单纯的聚合物 PCL- $b$-PEG- $b$-PCL 不能形 成多孔结构薄膜, 表面基本光滑(如图 2a 所示). 而在相 同条件下，以 $n(\mathrm{DEP} 5 \mathrm{~A}): n(\mathrm{PCL}-b-\mathrm{PEG}-b-\mathrm{PCL})=15$ : 1 , 浓度为 $c(\mathrm{PCL}-b-\mathrm{PEG}-b-\mathrm{PCL})=5.0 \mathrm{mg} / \mathrm{mL}$ 的 PPR 溶 液形成规整的呼吸图阵列(如图 $2 b$ 所示), 薄膜表面在白 光下显示出珍珠贝母色, 膜表面平均孔径为 $2.15 \mu \mathrm{m}$ (膜 表面孔径测量值的平均值, 测量个数 $\geqslant 30$ 个). 我们认 为这是由于纯聚合物 PCL- $b$-PEG- $b$-PCL 均为柔性链, 当水滴凝聚时, 聚合物溶液不足以维持沉积水滴的规则 排列, 水滴间发生融合和流动, 最终使得纯聚合物 PCL- $b$-PEG- $b$-PCL 不能形成多孔薄膜. 因此, 柱芳烃与 PCL 链段的络合能改变聚合物的链的原有性能, 使得聚 合物能发挥出更加优异的功能和性质.

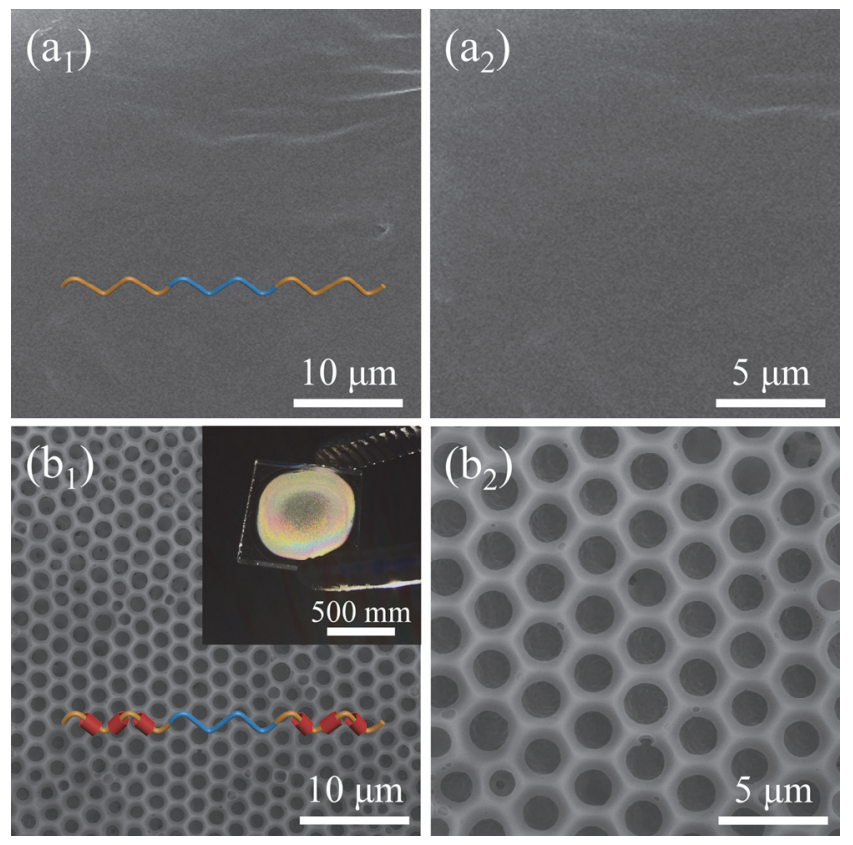

图 2 用(a) PCL- $b$-PEG- $b$-PCL $(5.0 \mathrm{mg} / \mathrm{mL})$ 和(b) PPR $(5.0 \mathrm{mg} / \mathrm{mL}$ PCL- $b$-PEG- $b$-PCL, 15.0 equiv. of DEP5A)在相同条件下(氯仿中, $90 \%$ $R H, 25{ }^{\circ} \mathrm{C}$ )制备得到的薄膜的 SEM 图. 图 $a_{2}$ 及图 $b_{2}$ 分别为图 $a_{1}$ 及图 $b_{1}$ 的放大图. 图 $b_{1}$ 的插入图为白光下薄膜的衍射图

Figure 2 SEM images of the films fabricated under $90 \% \mathrm{RH}$ at $25{ }^{\circ} \mathrm{C}$ using chloroform as the solvent. (a) PCL- $b$-PEG- $b$-PCL $(5.0 \mathrm{mg} / \mathrm{mL})$ and (b) PPR ( $5.0 \mathrm{mg} / \mathrm{mL}$ PCL- $b$-PEG- $b$-PCL, 15.0 equiv. of DEP5A). ( $\left.\mathrm{a}_{2}\right)$ and $\left(b_{2}\right)$ are the magnified pictures of $\left(a_{1}\right)$ and $\left(b_{1}\right)$, respectively. The inserted pictures of Figure $2 b_{1}$ is the photograph of the diffraction of the film under white light

我们进一步研究了 PPR 蜂窝状多孔薄膜的亲疏水 性. 如图 3a 所示, 将 8 滴用亚甲基蓝染色的水滴滴在多 孔薄膜上, 可以发现, 薄膜具有疏水性(接触角 $\left.>130^{\circ}\right)$, 这是由于当水滴滴在薄膜上时, 薄膜上的孔仍有大量的 空气，这些空气在液滴表面和固体表面充当气垫的作用 支撑水滴, 使得薄膜展现出疏水性. 利用薄膜的疏水性,
我们能在薄膜上实现水滴的图案化. 同时, 当薄膜 $90^{\circ}$ 放置，水滴也不会从薄膜流动下来，展现出玫瑰花瓣效 应. 这种具有疏水性和高粘附性的薄膜有望应用在微量 液体运输等领域.
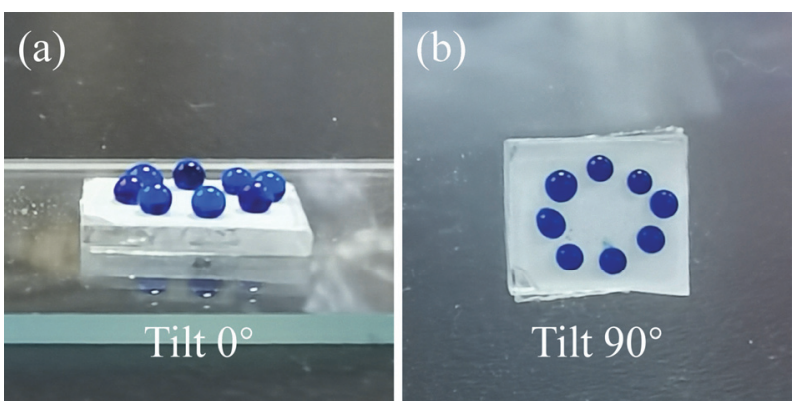

图 $310 \mu \mathrm{L}$ 水滴(亚甲基蓝染色)滴在 PPR 蜂窝状多孔薄膜上在倾斜 角为(a) $0^{\circ}$ 和(b) $90^{\circ}$ 时的照片

Figure 3 Photographs of $10 \mu \mathrm{L}$ water droplets (dyed by methylene blue) on PPR honeycomb porous film at (a) $0^{\circ}$ and (b) $90^{\circ}$

\subsection{2乙醇氛围下制备 PPR 球状组装体薄膜}

基于上述实验结果，我们探索了溶剂和柱芳烃物质 的量比对形成球状组装体薄膜的影响(图 S7, S8), 发现 在 $25{ }^{\circ} \mathrm{C}$ 和 $90 \% \mathrm{RH}$ 的条件下, 在不加入柱芳烃的情况 下，单纯的聚合物 PCL- $b$-PEG- $b$-PCL，只能形成不规则 组装体(如图 $4 \mathrm{a}$ 所示). 而在相同条件下, 以 $n$ (DEP5A) : $n(\mathrm{PCL}-b$-PEG- $b$-PCL $)=15: 1$, 浓度为 $c(\mathrm{PCL}-b-\mathrm{PEG}-$
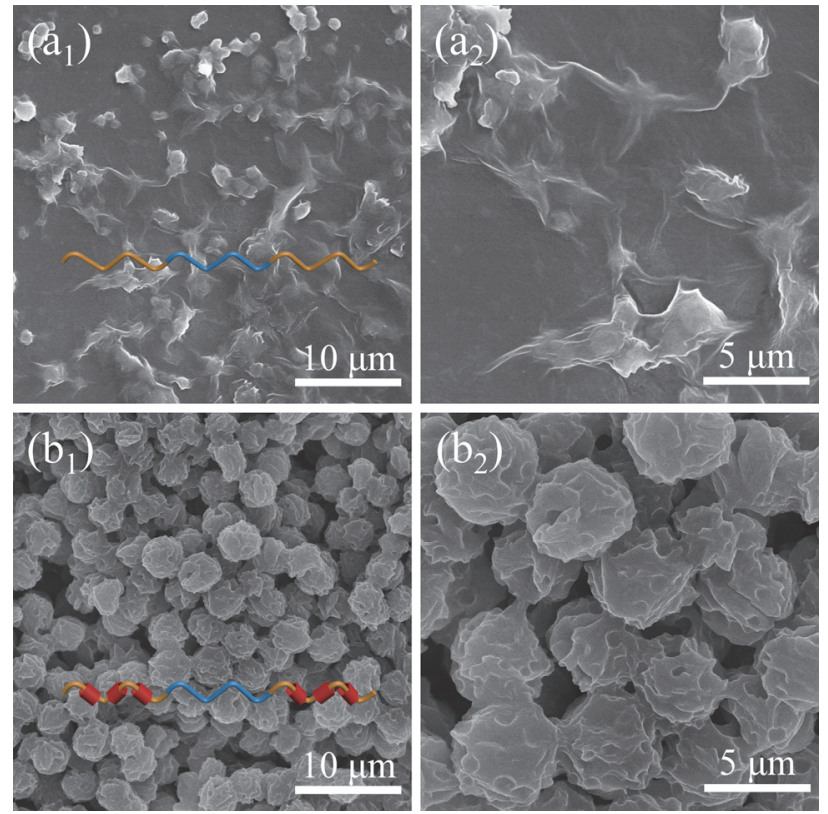

图 4 用(a) PCL- $b$-PEG- $b$-PCL $(5.0 \mathrm{mg} / \mathrm{mL}$ ) 和(b) PPR $(5.0 \mathrm{mg} / \mathrm{mL}$ PCL- $b$-PEG- $b$-PCL, 15.0 equiv. of DEP5A)在相同条件下(氯仿中, 乙醇 氛围, $25{ }^{\circ} \mathrm{C}$ 制备得到的薄膜的 SEM 图. 图 $a_{2}$ 及图 $b_{2}$ 分别为图 $a_{1}$ 及图 $\mathrm{b}_{1}$ 的放大图

Figure 4 SEM images of the films fabricated under ethanol atmosphere at $25{ }^{\circ} \mathrm{C}$ using chloroform as the solvent. (a) PCL- $b$-PEG- $b$-PCL (5.0 $\mathrm{mg} / \mathrm{mL})$ and (b) PPR $(5.0 \mathrm{mg} / \mathrm{mL}$ PCL- $b$-PEG- $b$-PCL, 15.0 equiv. of DEP5A). $\left(a_{2}\right)$ and $\left(b_{2}\right)$ are the magnified pictures of $\left(a_{1}\right)$ and $\left(b_{1}\right)$, respectively 
$b-\mathrm{PCL})=5.0 \mathrm{mg} / \mathrm{mL}$ 的 PPR 溶液形成规整的球状组装体 (如图 4b 所示), 并且球状组装体表面为层层褶皱的形 貌, 小球平均直径为 $3.34 \mu \mathrm{m}$ (膜表面粒径测量值的平均 值, 测量个数 $\geqslant 30$ 个).

\section{2 超分子薄膜的竞争性客体响应}

PPR 具有竞争性客体响应, 因此, 我们可以利用 PPR 的竞争性客体响应通过简单的浸泡法改变薄膜的 微结构及薄膜的润湿性. 如图 5 所示, 蜂窝状多孔薄膜 和球状组装体薄膜被浸泡在含竞争性客体 $\mathrm{DBrBu}$ 的乙 醇溶液中 $(20.0 \mu \mathrm{L} \mathrm{DBrBu}+10.0 \mathrm{~mL}$ 乙醇) 不同时间, 再 将薄膜取出并泡进乙醇溶液中 $30 \mathrm{~s}$ 以去除多余游离的 $\mathrm{DBrBu}$ 分子, 最后将薄膜取出, 并放置在常温下等待乙 醇挥发, 最终得到微结构改变的薄膜.

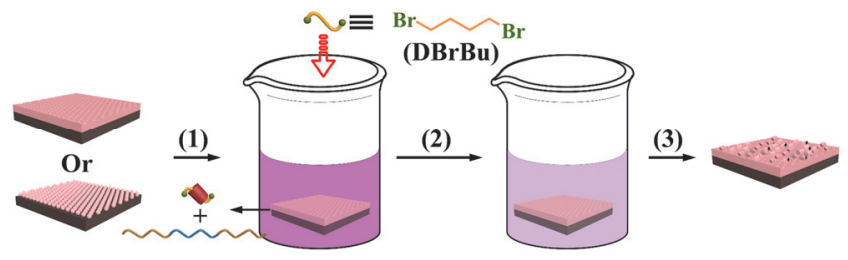

图 5 PPR 超分子薄膜竞争性客体响应实验流程示意图. (1)将薄膜放 入含竞争性客体 $\mathrm{DBrBu}$ 的乙醇溶液中 $(20.0 \mu \mathrm{L} \mathrm{DBrBu}+10.0 \mathrm{~mL}$ 乙醇 $)$ 一定时间; (2)将薄膜取出并泡进乙醇溶液中 $30 \mathrm{~s}$ 以去除多余游离的 $\mathrm{DBrBu}$ 分子; (3) 将薄膜取出, 并放置在常温下等待乙醇挥发, 最终得 到由片状组装体组成的薄膜

Figure 5 Schematic illustration of the process of competitive guest response experiment of PPR supramolecular films. (1) The films were immersed in $\mathrm{DBrBu}$-containing ethanol solution $(20.0 \mu \mathrm{L} \mathrm{DBrBu}+10.0$ $\mathrm{mL}$ ethanol) for different times; (2) the films were taken out and immersed in ethanol solution to remove excess $\mathrm{DBrBu}$; (3) the films were taken out and dried under room temperature
浸泡不同的时间，我们分别获得了不同形貌的薄 膜. 如图 6 所示，随着浸泡在含竞争性客体溶液的时间 的增长, 规整的多孔结构和球状组装体结构逐步瓦解. 如图 6a 所示, 当浸泡时间为 $30 \mathrm{~s}$ 时, 多孔结构变得不规 整, 薄膜表面出现小于 $1 \mu \mathrm{m}$ 的片状组装体. 随着浸泡 时间增加到 $2 \mathrm{~h}$, 多孔结构完全消失, 出现了大量的片 状或块状组装体, 这是由于 $\mathrm{DBrBu}$ 与 DEP5A 能形成更 强的主客体作用, DEP5A 从 PCL 链段上脱落下来, 并与 $\mathrm{DBrBu}$ 形成了准轮烷. 该准轮烷有高度的结晶性, 在合 适温度下, 最终形成了片状组装体. 这种表面微结构的 改变, 使得薄膜润湿性质发生明显改变, 从最初的多孔 结构时薄膜水接触角为 $135.1^{\circ}$, 到浸泡时间 $30 \mathrm{~s}$ 后水接 触角为 $110.8^{\circ}$, 到最后多孔结构瓦解后水接触角为 $89.7^{\circ}$. 相同地, 球状组装体先经历溶胀到瓦解的过程 (如图 $6 b_{1-3}$ ), 薄膜的水接触角变化从 $133.2^{\circ}$ 降为 $112.8^{\circ}$.

为进一步探究 PPR 超分子薄膜的竞争性客体响应 对薄膜性质的影响, 我们测量了多孔薄膜和球状组装体 薄膜在浸泡不同时间后的水接触角，设置 5 组浸泡相同 时间，最终得到图 7 所示薄膜接触角与浸泡竞争性客体 溶液时间的关系. 随着浸泡时间的延长, 薄膜接触角都 会明显下降. 在浸泡 $2 \mathrm{~h}$ 后, 多孔薄膜的水接触角下降 了约 33\%, 球状组装体薄膜下降了约 $15 \%$. 这些结果证 明了 PPR 超分子薄膜具有竞争性客体响应和通过简单 的浸泡方法可调控薄膜的润湿性.

\section{3 结论}

本文利用具有竞争性客体响应的 PPR 通过呼吸图 法制备了蜂窝状多孔薄膜和球状组装体薄膜. 通过与纯
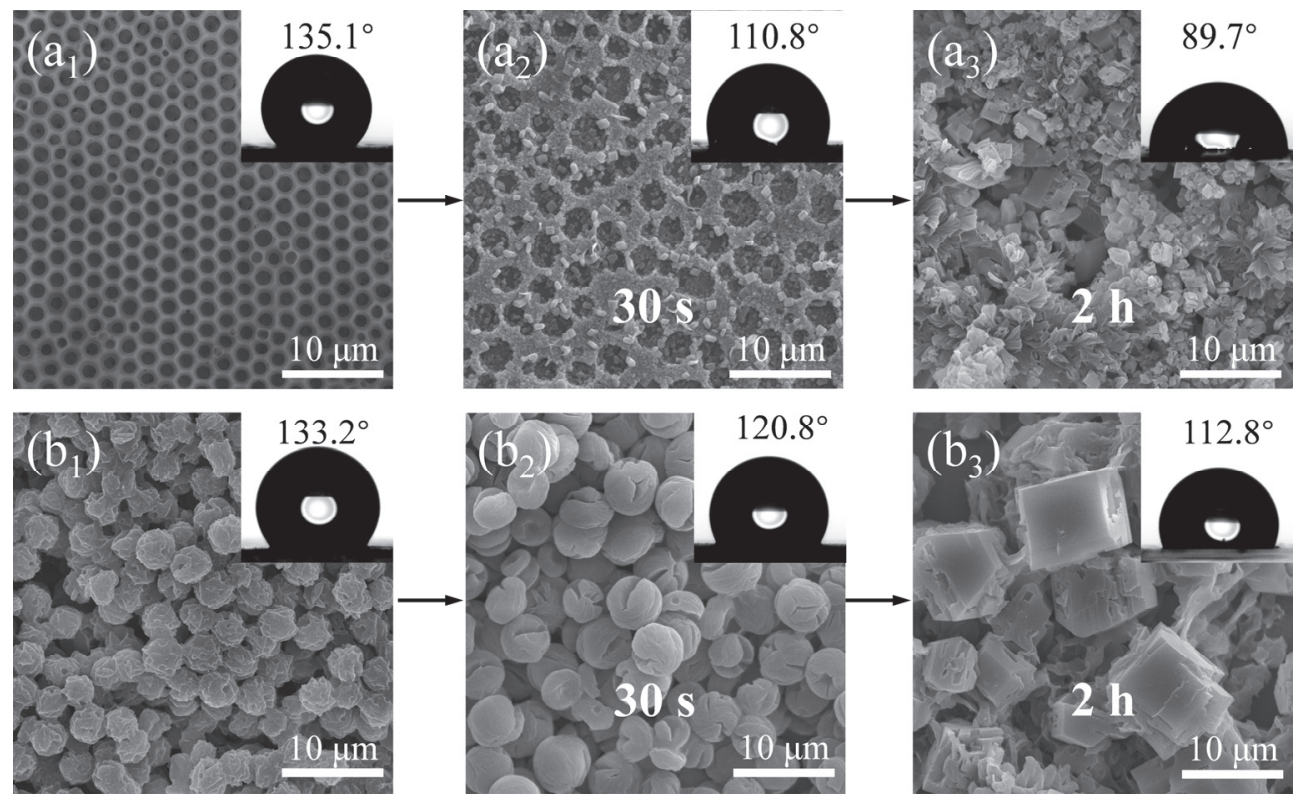

图 6 PPR 蜂窝状多孔薄膜和球状组装体薄膜浸泡在含 $\mathrm{DBrBu}$ 的乙醇溶液不同时间后表面形貌的 SEM 图及水接触角

Figure 6 The SEM images and the water contact angles of PPR honeycomb porous films and sphere films after being immersed in DBrBu-containing ethanol solution for different times 

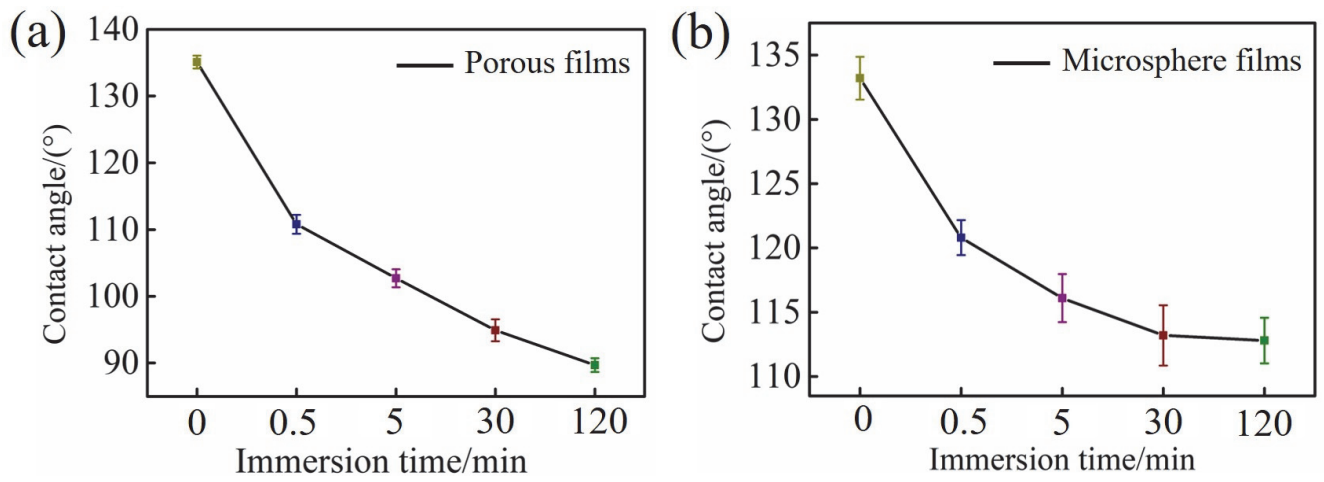

图 7 (a) 蜂窝状多孔薄膜和(b) 球状组装体薄膜浸泡在含 $\mathrm{DBrBu}$ 的乙醇溶液不同时间后薄膜的水接触角变化图

Figure 7 The water contact angle curves of (a) honeycomb porous films and (b) sphere films after being immersed in DBrBu-containing ethanol solution for different times

聚合物对比发现, DEP5A 的加入改善了聚合物成膜的性 质, 在 $25{ }^{\circ} \mathrm{C}$ 和 $90 \% \mathrm{RH}$ 或乙醇氛围的条件下, 以 $n$ (DEP5A) : $n$ (PCL- $b$-PEG- $b$-PCL $)=15: 1$, 浓度为 $c(\mathrm{PCL}-b-\mathrm{PEG}-b-\mathrm{PCL})=5.0 \mathrm{mg} / \mathrm{mL}$ 的 PPR 溶液能形成规 整的蜂窝状多孔膜或球状组装体薄膜. 由于薄膜的规整 性及其微结构, 薄膜具有明显的疏水性和高粘附性. 进 一步, 通过简单的浸泡法, 可改变薄膜的微结构及其水 接触角, 实现了对薄膜润湿性的控制与改变. 我们相信, 本文的研究工作对于新型刺激响应性智能薄膜研究具 有重要的参考价值.

\section{4 实验部分}

\section{1 试剂}

二氯甲烷、氯仿、二硫化碳、四氢呋喃、乙醇、1,4二溴丁烷等购自上海泰坦科技有限公司, 试剂纯度为分 析纯. 1,4-二乙氧基柱[5]芳烃(DEP5A)和 PCL- $b$-PEG$b$-PCL 根据本课题组已发表的文献制备 ${ }^{[43]}$.

\section{2 呼吸图法制备聚合物多孔膜}

称取 $5.0 \mathrm{mg}$ 的 PCL- $b$-PEG- $b$-PCL 嵌段聚合物和适 量的 DEP5A 溶解于 $1 \mathrm{~mL}$ 相应的溶剂中, 配制成不同柱 芳烃物质的量比(5、10、15、20、30 equiv.)的 PPR 溶液. 在恒温恒湿箱中, 设置相应的温度和湿度, 将 $20 \mu \mathrm{L}$ 配 制好的聚合物溶液滴在洁净的 $0.8 \mathrm{~cm} \times 0.8 \mathrm{~cm}$ 的玻璃片 上, 待溶剂完全挥发后, 得到白色的聚合物薄膜.

在乙醇氛围中制备聚合物薄膜时, 向带有磨口瓶塞 的 $50 \mathrm{~mL}$ 玻璃广口瓶中放入一个带支架的塑料平板, 并 在平板上放置洁净的玻璃片, 加入 $10 \mathrm{~mL}$ 乙醇, 密封 30 $\min$, 使得瓶内乙醇气氛达到饱和. 在玻璃片上滴加 20 $\mu \mathrm{L}$ 配置好的聚合物溶液并盖好瓶塞. 静置数 $10 \mathrm{~min}$, 待 溶剂完全挥发后, 取出玻璃片得到白色聚合物薄膜.

\section{3 分析表征与仪器}

扫描电子显微镜(SEM)用于观察聚合物膜表面形 貌，生产厂家: HITACHI，规格型号: S4800.
接触角测量仪用于测量薄膜表面水滴的接触角，文 中接触角数据为测试 10 次后取得的平均值, 生产厂家: Dataphysics.

\section{References}

[1] Zhang, A.; Bai, H.; Li, L. Chem. Rev. 2015, 115, 9801.

[2] Yabu, H.; Jia, R.; Matsuo, Y.; Ijiro, K.; Yamamoto, S.; Nishino, F.; Takaki, T.; Kuwahara, M.; Shimomura, M. Adv. Mater. 2008, 20, 4200.

[3] Lan, P.; Li, J.; Gong, J.; Li, L. Acta Chim. Sinica 2012, 70, 45 (in Chinese). (兰平, 李剑, 龚剑亮, 李否, 化学学报, 2012, 70, 45.)

[4] Geng, F.; Chen, J.; Zhao, Q.; Li, J.; Ma, Z. Acta Chim. Sinica 2011, 69, 2741 (in Chinese). (耿风华, 陈健壮, 赵巧玲, 李剑, 马志, 化 学学报, 2011, 69, 2741.)

[5] Yang, X.-Y.; Chen, L.-H.; Li, Y.; Su, B.-L. Chem. Soc. Rev. 2017, 46, 481.

[6] Wu, Y.; Shah, D. U.; Wang, B.; Liu, J.; Ren, X.; Ramage, M. H.; Scherman, O. A. Adv. Mater. 2018, 30, 1707169.

[7] Yang, Y.; Li, X.; Zheng, X.; Chen, Z.; Zhou, Q.; Chen, Y. Adv. Mater. 2018, 30, 1704912.

[8] Xu, Y. Y.; Wang, W.; Chen, J. Z.; Lin, S. L. Chin. J. Org. Chem. 2018, 38, 2161 (in Chinese). (徐悦莹, 王伟, 陈健壮, 林绍梁, 有 机化学, 2018, 38, 2161.)

[9] Heng, L.; Guo, T.; Wang, B.; Fan, L.-Z.; Jiang, L. J. Mater. Chem. A 2015, 3, 23699.

[10] Li, C.; Zhang, Y.; Ju, J.; Cheng, F.; Liu, M.; Jiang, L.; Yu, Y. $A d v$. Funct. Mater. 2012, 22, 760.

[11] Chen, Y.; Li, F.; Cao, W.; Li, T. J. Mater. Chem. A 2015, 3, 16934.

[12] Ma, J.; Yan, H.; Quan, J.; Bi, J.; Li, H. ACS Appl. Mater. Interfaces 2019, $11,1665$.

[13] Ding, J.; Zhang, A.; Li, L. Soft Matter 2013, 9, 506.

[14] Li, H.; Yang, Y.; Xu, F.; Liang, T.; Wen, H.; Tian, W. Chem. Commun. 2019, 55, 271.

[15] Wang, X.-H.; Song, N.; Hou, W.; Wang, C.-Y.; Wang, Y.; Tang, J.; Yang, Y.-W. Adv. Mater. 2019, 31, 1903962.

[16] Li, X.-S.; Li, Y.-F.; Wu, J.-R.; Lou, X.-Y.; Han, J.; Qin, J.; Yang, Y.-W. J. Mater. Chem. A 2020, 8, 3651.

[17] Chen, J.; Meng, G.; Zhu, Q.; Zhang, S.; Chen, P. J. Mater. Chem. C 2019, 38, 11747.

[18] Catalan, A.; Tiburcio, J. Chem. Commun. 2016, 52, 9526.

[19] Steck, J.; Yang, J.; Suo, Z. ACS Macro Lett. 2019, 8, 754.

[20] Wu, C.-H.; Lu, C.-S.; Chen, W.-L.; Tung, S.-H.; Jeng, R.-J. Macromol. Mater. Eng. 2018, 303, 1700433.

[21] Li, J.; Han, Y.; Chen, C. F. Chin. J. Org. Chem. 2020, 40, 3714 (in Chinese). (李晶, 韩莹, 陈传峰，有机化学, 2020, 40, 3714.)

[22] Bertrand, A.; Bousquet, A.; Lartigau-Dagron, C.; Billon, L. Chem. Commun. 2016, 52, 9562 .

[23] Wu, X.; Duan, Q. P.; Ni, M. F.; Hu, X. Y.; Wang, L. Y. Chin. J. Org. Chem. 2014, 34, 437 (in Chinese). (吴旋, 段群鹏, 倪梦飞, 胡晓 玉, 王乐勇, 有机化学, 2014, 34, 437.) 
[24] Luo, L.; Nie, G.; Tian, D.; Deng, H.; Jiang, L.; Li, H. Angew. Chem. Int. Ed. 2016, 55, 12713.

[25] Tang, X.; Tang, X. Z.; You, Y.; Ren, L.; Wang, Y.; Yan, L. Acta Chim. Sinica 2012, 70, 1565 (in Chinese). (唐翔, 唐先忠, 游英才, 任立轫, 王洋, 严立京, 化学学报, 2012, 70, 1565.)

[26] Ogoshi, T.; Yoshikoshi, K.; Aoki, T.; Yamagishi, T. Chem. Commun. 2013, 49, 8785.

[27] Fasano, V.; Baroncini, M.; Moffa, M.; Iandolo, D.; Camposeo, A.; Credi, A.; Pisignano, D. J. Am. Chem. Soc. 2014, 136, 14245.

[28] Gao, P.; Wang, P.; Geng, X.; Ye, L.; Zhang, A.; Feng, Z. Acta Chim. Sinica 2013, 71, 347 (in Chinese). (高鹏, 王培境, 耿雪, 叶霖, 张 爱英, 冯增国, 化学学报, 2013, 71, 347.)

[29] Pan, S.; Ni, M.; Mu, B.; Li, Q.; Hu, X.-Y.; Lin, C.; Chen, D.; Wang, L. Adv. Funct. Mater. 2015, 25, 3571.

[30] Ahn, Y.; Jang, Y.; Selvapalam, N.; Yun, G.; Kim, K. Angew. Chem. Int. Ed. 2013, 52, 3140 .

[31] Ghasemi, S.; Besharati, M. Polym. Adv. Technol. 2020, 31, 3104.

[32] Li, Y.; Ma, X.; Ma, J.; Zhang, Z.; Niu, Z.; Chen, F. Polymers 2021, $13,316$.

[33] Ma, C.; Zhong, Y.; Li, J.; Chen, C.; Gong, J.; Xie, S.; Li, L.; Ma, Z. Chem. Mater. 2010, 22, 2367.

[34] Du, C.; Zhang, A.; Bai, H.; Li, L. ACS Macro Lett. 2013, 2, 27.
[35] Escalé, P.; Camp, W. V.; Prez, F. D.; Rubatat, L.; Billon, L.; Save, M. Polym. Chem. 2013, 4, 4710.

[36] Zander, N. E.; Orlicki, J. A.; Karikari, A. S.; Long, T. E.; Rawlett, A. M. Chem. Mater. 2007, 19, 6145.

[37] Yabu, H.; Hirai, Y.; Kojima, M.; Shimomura, M. Chem. Mater. 2009, $21,1787$.

[38] Muller, M.; Zentel, R.; Maka, T.; Romanov, S. G.; Torres, C. S. $A d v$. Mater. 2000, 12, 1499.

[39] Wang, W.; Du, C.; Wang, X.; He, X.; Lin, J.; Li, L.; Lin, S. Angew. Chem. Int. Ed. 2014, 53, 12116.

[40] Wang, W.; Yao, Y.; Luo, T.; Chen, L.; Lin, J.; Li, L.; Lin, S. ACS Appl. Mater. Interfaces 2017, 9, 4223.

[41] Wang, W.; Shen, D.; Li, X.; Yao, Y.; Lin, J.; Wang, A.; Yu, J.; Wang, Z. L.; Hong, S. W.; Lin, Z.; Lin, S. Angew. Chem. Int. Ed. 2018, 57 , 2139.

[42] Gao, F.; Yao, Y.; Wang, W.; Wang, X.; Li, L.; Zhuang, Q.; Lin, S. Macromolecules 2018, 51, 2742.

[43] Li, J.; Dong, J.; Cui, K.; Wang, H.; Sun, Y.; Yao, Y.; Chen, J.; Gu, J.; Lin, S. J. Mater. Chem. A 2020, 8, 10917.

[44] Wan, L. S.; Zhu, L. W.; Ou, Y.; Xu, Z. K. Chem. Commun. 2014, 50, 4024 .

(Cheng, B.) 